\title{
Paraumbilical Perforator Flap: A Good Choice for the Repair of the Deep Soft Tissue Defects in the Hand and Forearm
}

\author{
Jin Wang ${ }^{1,2}$, Min Wang ${ }^{1}$, Yuan $\mathrm{Xu}^{2}$, Yao Guo ${ }^{2}$, Lei Cui ${ }^{2}$, Jun Wang ${ }^{2}$, Zhi-Jian Hong ${ }^{2}$ and Si-Ming Yuan ${ }^{2^{*}}$ \\ ${ }^{1}$ Department of Burn and Plastic Surgery, Nanjing Gulou Hospital Suqian City People's Hospital Group, China \\ ${ }^{2}$ Department of Burn and Plastic Surgery, Jinling Hospital, School of Medicine, Nanjing University, China
}

*Corresponding author: Si-Ming Yuan, Department of Burn and Plastic Surgery, Jinling Hospital, School of Medicine, Nanjing University, 305 East Zhongshan Road, Nanjing 210002, Jiangsu, China, Tel: 086-25-80860020; E-mail: yuansm@163.com

Received date: October 10, 2017, Accepted date: October 12, 2017, Published date: October 16, 2017

Copyright: (c) 2017 Wang J, et al. This is an open-access article distributed under the terms of the Creative Commons Attribution License, which permits unrestricted use, distribution, and reproduction in any medium, provided the original author and source are credited.

\begin{abstract}
Objective: Severe trauma to the hand or forearm often results in the deep soft tissue defect with the exposure of tendon or bone, which needs to be repaired by skin flaps. In this study, we introduced the use of paraumbilical perforator flap in the repair of such defects, and discussed its advantages and disadvantages.

Methods: From Jan. 2006 to Dec. 2016, 14 patients with deep soft tissue defects in the hand and forearm were admitted into our clinic. The defects were caused by hot-crush injury $(n=2)$, electrical injury $(n=4)$, severe burn $(n=2)$, crush injury $(n=3)$, and avulsion injury $(n=3)$. After admission, the patients underwent the debridement, vacuum suction, and skin graft to repair partial wound. The deep defects with the exposure of tendon and bone were repaired by paraumbilical perforator flap finally.
\end{abstract}

Results: All of the 14 flaps survived after the transfer in the first-stage operation. The width of the flap's pedicle ranged from $6 \mathrm{~cm}$ to $8 \mathrm{~cm}$, and the length ranged from $16 \mathrm{~cm}$ to $20 \mathrm{~cm}$. 1620 days later, the second-stage operation was done to divide the pedicle. After the division, $11 / 14$ of the flaps survived well. The remaining $3 / 14$ flaps had the partial necrosis of the margin, which healed with changing dressing. In the follow-up period, most of the flaps had satisfying appearance. 2 flaps were bulky and thinned by surgery.

Conclusions: The paraumbilical perforator flap has reliable blood supply, good texture, sufficient area, and is convenient to transfer. It's an ideal choice for the repair of the deep soft tissue defect in the hand and forearm.

Keywords: Paraumbilical perforator flap; Hand; Forearm; Deep soft tissue defect

\section{Introduction}

Severe trauma to the hands or forearms, such as electrical injuries, crush injuries, and deep burns, often result in deep defects of skin and soft tissues, and the exposure of tendon or bone, which need the repair by flaps, including the abdominal pedicle flaps. Most of the abdominal pedicle flaps were the random flaps so that their application was restricted by flap's length/width ratio and the transfer angle [1]. In 1983, Taylor and others first reported the use of the paraumbilical perforator flap [2], which is a type of axial vascular flap supplied by the perforator vessels beside the umbilicus. The paraumbilical perforator flap has many advantages, such as reliable blood supply, good texture, sufficient area, and convenient transfer [3-6]. In this report, we reviewed the use of this flap in repairing the deep defect in the hands and arms in the past ten years in our clinic, and discussed its advantages and disadvantages.

\section{Patients and Methods}

This research was approved by the Committee on Clinical Investigation of Jinling Hospital. Informed consent was provided for the patients' parents or guardian, according to the Declaration of Helsinki. From January 2006 to December 2016, 14 patients (10 males and 4 females) with deep soft tissue defects in the hands and forearms were included in this study. The patients' age ranged from 21 to 57 years, with the average age 33.5 years. The defects were caused by hotcrush injury $(n=2)$, electrical injury $(n=4)$, severe burn $(n=2)$, crush injury $(n=3)$, and avulsion injury $(n=3)$. All of the patients had deep soft tissue defects accompanied by the exposure of tendon or bone (Figure 1).

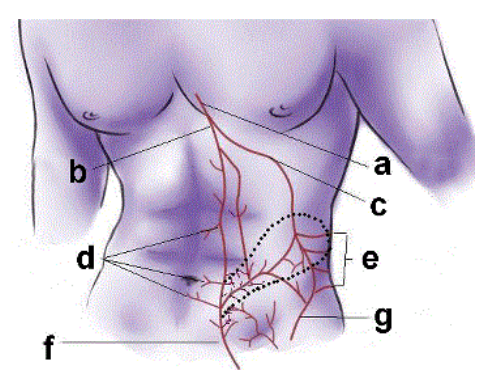

Figure 1: The design of the paraumbilical perforator flap (a, b, c, d, $\mathrm{e}, \mathrm{f}$, and $\mathrm{g}$ mean the internal mammary artery, superior epigastric artery, musculophrenic artery, periumbilical perforating arteries, subcostal and lumber arteries, inferior epigastric artery, and ascending branch of deep circumflex artery, respectively). 
After admission, each patient's general condition was assessed by routine blood tests, blood biochemistry examination, coagulation function examination, electrocardiography, chest radiography, and other necessary examinations.

The debridement of the wounds was performed and the negative pressure wound therapy (NPWT) was used in some cases to accelerate the vascularization of the wound. Skin graft was used to repair partial wound. The deep defects with the exposure of tendon and bone were repaired by paraumbilical perforator flap finally (Figure 2).
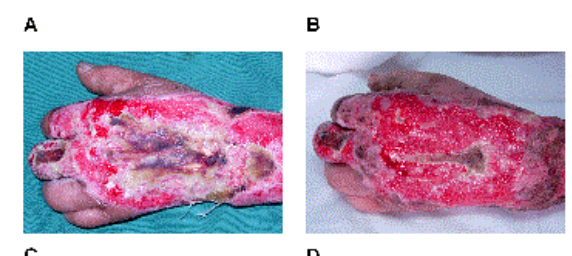

c D

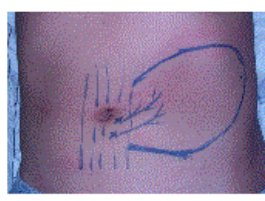

E
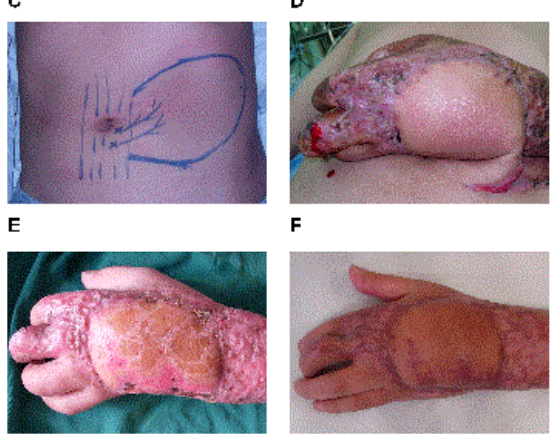

Figure 2: Case 1-(A) A 50-year-old man had wounds on the forearm and hand caused by hot-crush injury, with the exposure of necrotic tendon and the second proximal phalanx. (B) Debridement, changing of the dressing, and skin graft were performed to repair the partial wound on the forearm. The third metacarpal bone was exposed in the wound. (C) A left paraumbilical perforator flap was designed. (D) The flap was formed and transferred to cover the wound. (E) Twenty days later, the pedicle of the flap was divided. (F) One year later, the flap had an acceptable appearance.

In the design of flap, a Doppler blood vessel detection instrument was used to determine the routes of the inferior epigastric artery and its perforating branches. The major paraumbilical branch was located at the point $3 \mathrm{~cm}$ beneath the umbilicus and $2 \mathrm{~cm}$ from the central line, with higher sound of blood flow.

This was used as the start point of the vascular pedicle of the flap, and marked with the methylene. Then a line was drawn between this point and the angulus inferior scapulae point, which was the axial line of the flap. So the flap was designed along this line according to shape and area of the defect in the hand and forearm. The width of the pedicle ranged from $6 \mathrm{~cm}$ to $8 \mathrm{~cm}$, and the length of the flap ranged from $16 \mathrm{~cm}$ to $20 \mathrm{~cm}$.

In the operation, the debridement was performed again. The edema granulation tissue was cleared. Tendons, nerves, blood vessel bundles, and bone were kept. The wound was cleaned with hydrogen peroxide, iodophor, and normal saline. After that, the design of flap was adjusted again to adapt to the wound. The flap was then formed from the distal end to the pedicle (Figure 3).

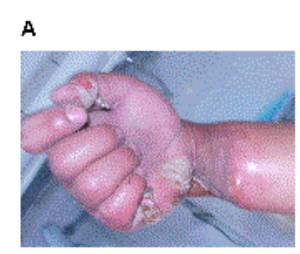

c

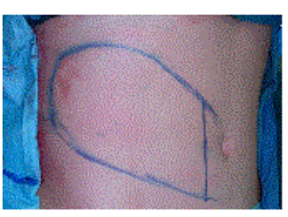

E

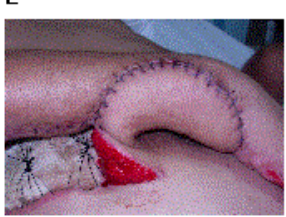

B
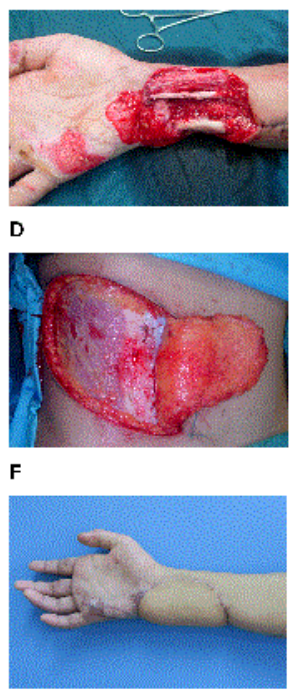

Figure 3: Case 2-(A) A 25-year-old man had a severe electrical injury on the right forearm and hand. (B) Debridement and changing of the dressing were done. The tendon and ulna were exposed in the wound. (C) A right paraumbilical perforator flap was designed. (D) The flap was formed. (E) The flap was transferred to cover the wound. (F) Twenty days later, the pedicle of the flap was divided. The flap survived very well.

The separation stopped at the lateral border of the rectus abdominis epitheca. The pedicle was adjusted to facilitate the transfer of flap. Before the transfer, skin grafting was performed to cover the wounds in the abdominal wall. Then the flap was transferred from the abdomen to the forearm or hand to repair the wounds. The gauze was placed under or outside the flap to make the flap fit the wounds exactly. The upper limb was then fixed to the abdominal wall with an elastic bandage. After the operation, the dressing was changed every day to observe the survival of the flap and clear the secretions. Antibiotics were used to prevent the infection. 12 to 14 days later, the block test of the blood supply of the pedicle was made. 16 to 21 days later, the pedicle was divided. The patient's dressing was changed regularly until the wounds healed. The post-discharge follow-up period lasted 4 months to 2 years.

\section{Results}

All of the 14 flaps survived after transfer in the first-stage operation. The mean pedicle width was $6 \mathrm{~cm}$ to $8 \mathrm{~cm}$, the mean length of the flap was $16 \mathrm{~cm}$ to $20 \mathrm{~cm}$, and the average time until pedicle division was 1620 days. After division, 11/14 of the flaps survived well. The remaining 3/14 flaps showed partial necrosis of the margins, which healed with several dressing changes. During the follow-up period, most of the flaps had a satisfying appearance. Two flaps were bulky and were thinned by surgery.

\section{Discussion}

As the hands and forearms are generally exposed, they have a high probability of injury. Severe trauma to the hands or forearms, such as electrical injuries, hot-crush injuries, crush injuries, and deep burns, 
often result in deep defects of the soft skin tissues and exposure of tendon or bone. Such wounds are difficult to repair and severely impact the functions of the hand and forearm. Skin graft on such wounds can't survive well and achieve good appearance and function. The abdominal pedicle flaps are often used in such cases, most of which are random flaps. However, the application of random flap is limited by the length/width ratio, swollen pedicle, and inconvenient transfer.

The paraumbilical perforator flap is a type of axial vascular flap in which perforator vessels beside the umbilicus are used as nutritional vessels. This flap has reliable blood supply. Its length/width ratio can be up to $4: 1$, which is obviously higher than that of the traditional abdominal random flap. The design of flap is flexible and the transfer is very convenient [7]. The distal part of the flap can be trimmed to create a thin flap to get better color and texture [8]. The donor sites in some cases can be sutured directly without skin grafting. The above advantages show that the paraumbilical perforator flap is suitable for repairing deep defects in soft skin tissues on the hand and forearms $[9,10]$.

In the operation, the separation should be very carefully and the perforating branches must be kept as more as possible, which are beneficial for the blood supply of flap. After the operation, the reliable bandage should be made to fit the flap to the wound. The forearm and hand must be fixed firmly on the abdominal wall; otherwise, the patient may draw out the arm due to discomfort, which results in the tearing of the flap. Analgesia is necessary to relieve the pain and improve the patient's rest, which helps the postoperative recovery and flap survival.

\section{Conclusion}

The results of our cases showed that the paraumbilical perforator flap is a good method for repairing deep defects in skin and soft tissues in the hands and forearms. However, this flap can't repair the tendon or bone defects in some cases. For them, the second-stage surgery of tendon or bone transplantation was needed to recover the function of hand and forearm.

\section{Acknowledgement}

This work was supported by the National Natural Science Foundation of China (No. 81272989) and the Science Foundation of Jinling Hospital (No. YYZD2014002).

\section{References}

1. Zang M, Zhu S, Song B, Jin J, Liu D, et al. (2012) Reconstruction of extensive upper extremity defects using pre-expanded oblique perforatorbased paraumbilical flaps. Burns 38: 917-923.

2. Taylor GI, Corlett R, Boyd JB (1983) The extended deep inferior epigastric flap: A clinical technique. Plast Reconstr Surg 72: 751-764.

3. Shukla L, Taylor GI, Shayan R (2013) The pedicled inferior paraumbilical perforator (I-PUP) flap for a volar wrist defect a reconstructive solution across the ages. Plast Reconstr Aesthet Surg 66: 1613-1615.

4. Yilmaz S, Saydam M, Seven E, Ercocen AR (2005) Paraumbilical perforator-based pedicled abdominal flap for extensive soft-tissue deficiencies of the forearm and hand. Ann Plast Surg 54: 365-368.

5. Seitchik SH, Granick MS, Solomon MP, Berman AT (1992) Post traumatic upper extremity wound coverage utilizing the extended deep inferior epigastric flap. Ann Plast Surg 28: 465-471.

6. GoushehJ, ArastehE, Mafi P (2008) Super-thin abdominal skin pedicle flap for the reconstruction of hypertrophic and contracted dorsal hand burn scars. Burns 34: 400-405.

7. O’Shaughnessy KD, Rawlani V, Hijjawi JB, Dumanian GA (2010) Oblique pedicled paraumbilical perforatorebased flap for reconstruction of complex proximal and mid-forearm defects: A report of two cases. Hand Surg Am 35: 1105-1110.

8. Koshima I, Moriguchi T, Soeda S, Tanaka H, Umeda N (1992) Free thin paraumbilical perforator-based flaps. Ann Plast Surg 29: 12-17.

9. Kamath BJ, Verghese T, Bhardwaj P (2007) "Wing flaps": Perforator-based pedicled paraumbilical flaps for skin defects in hand and forearm. Ann Plast Surg 59: 495-500.

10. Murakami M, Hyakusoku H (2004) Reconstruction of a defect after harvesting a radial forearm flap using a narrow pedicled paraumbilical perforator-based subdermal vascular network (super-thin) flap. Scand J Plast Reconstr Surg Hand Surg 38: 281-283. 